\title{
Design, Identification and Experimental Testing of a Light-weight Flexible-joint Arm for Aerial Physical Interaction
}

\author{
Burak Yüksel $^{1}$, Saber Mahboubi ${ }^{1}$, Cristian Secchi ${ }^{2}$, Heinrich H. Bülthoff ${ }^{1}$ and Antonio Franchi ${ }^{3,4}$
}

\begin{abstract}
In this paper we introduce the design of a lightweight novel flexible-joint arm for light-weight unmanned aerial vehicles (UAVs), which can be used both for safe physical interaction with the environment and it represents also a preliminary step in the direction of performing quick motions for tasks such as hammering or throwing. The actuator consists of an active pulley driven by a rotational servo motor, a passive pulley which is attached to a rigid link, and the elastic connections (springs) between these two pulleys. We identify the physical parameters of the system, and use an optimal control strategy to maximize its velocity by taking advantage of elastic components. The prototype can be extended to a light-weight variable stiffness actuator. The flexible-joint arm is applied on a quadrotor, to be used in aerial physical interaction tasks, which implies that the elastic components can also be used for stable interaction absorbing the interactive disturbances which might damage the flying system and its hardware. The design is validated through several experiments, and future developments are discussed in the paper.
\end{abstract}

\section{INTRODUCTION}

The physical interaction of flying robots, a.k.a. aerial physical interaction, has been a growing interest of the scientists and engineers in the last decade. The control of flying robots during physical interaction has become a challenge in terms of designing new tools or actuators, as well as developing powerful algorithms to allow the exertion of forces and torques on the environment while stabilizing the overall system and protect the expensive hardware.

There are various algorithms presented for aerial physical interaction. In [1], [2] an interconnection and damping assignment passivity-based controller is presented, where the algorithm assigns desired physical properties to the flying robot (quadrotor) depending on the force and torques coming from the environment. In [3], [4] passive decomposition methods have been designed for controlling the trajectory of a rigid tool onboard of a quadrotor. Cartesian impedance control for a manipulator placed on an UAV is shown in [5]. An impedance controller for aerial manipulation performed by a ducted-fan vehicle is presented in [6].

In parallel, other studies presented useful tools for improving the performance of physical interaction tasks. In [7], a light-weight industrial arm is attached to a small-size helicopter, under the consortium of [8]. Smaller scale designs are also presented in the literature. In [9] a 2D rigid arm

\footnotetext{
${ }^{1}$ Max Planck Institute for Biological Cybernetics, Spemanstr. 38, 72076, Tübingen, Germany. \{burak . yueksel, hhb\}@tuebingen.mpg. de

${ }^{2}$ DISMI-University of Modena and Reggio Emilia, Via G. Amendola 2, Morselli Building, 42122 Reggio Emilia Italy. cristian. secchi@unimore.it

${ }^{3}$ CNRS, LAAS, 7 avenue du colonel Roche, F-31400 Toulouse, France ${ }^{4}$ Univ de Toulouse, LAAS, F-31400 Toulouse, France afranchi@laas.fr
}

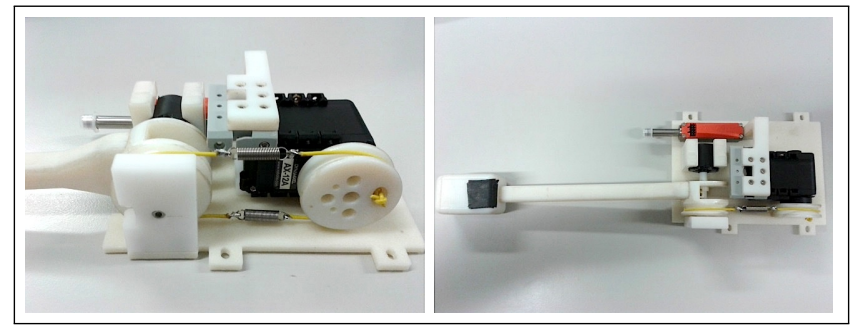

Fig. 1: The light-weight flexible-joint arm.

for aerial manipulation is introduced. A tool for surface inspection using a flying robot is developed and presented in [10]. Besides many different joints and actuated tools, passive ones are also used for physical interaction, as in [11].

In aerial physical interaction, it is necessary to always guarantee a safe (i.e., non-destabilizing) behavior of the system during any contact with the environment, that can be either desired or unforeseen (e.g., the robot moving in an unknown and/or hostile area). Furthermore, it should be possible to implement explosive motions for useful aerial interaction like aerial repairing or fixing. The use of flexible joints has proven to be successful for the implementation of interactive tasks when using grounded robotic arms or humanoid robots. The elasticity of the joints can in fact be exploited for achieving an intrinsic safe behavior of the system and for amplifying the mechanical performance of a rigid arm by exploiting the energy stored into the elastic element [12]. To the best of the authors' knowledge, the possibility of exploiting the benefits of elastic joints in aerial interaction has not been explored yet. The goal of this paper is to conduct a preliminary work to start filling this gap. We present a novel design of a light weight flexible-joint arm that can be mounted on a small size aerial vehicle for achieving an intrinsically safe aerial physical interaction and that allows to exploit the joint elasticity for implementing aerial explosive tasks (e.g., aerial hammering and aerial throwing). The proposed arm is then mounted on a quadrotor and its benefits during aerial physical interaction are experimentally validated.

The paper is organized as follows: in Section II we present the mechanical design and the mathematical modeling of the flexible-joint arm. The parameters of the linear models for both servo motor and flexible-joint arm dynamics are found by system identification methods and validated using experimental data. Section III is dedicated to the control framework of both flexible-joint arm and quadrotor, separately. The 


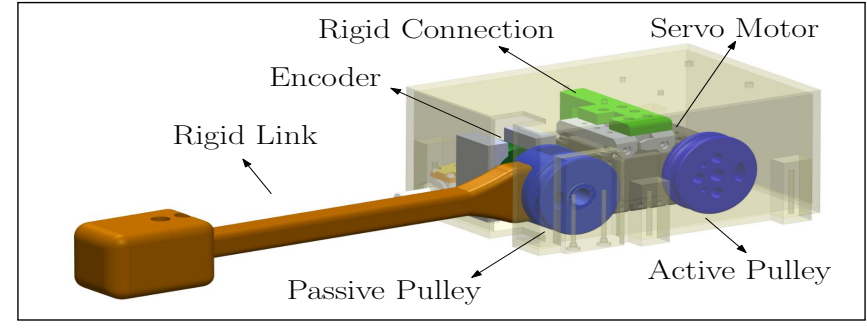

Fig. 2: CAD Model of the flexible-joint arm.

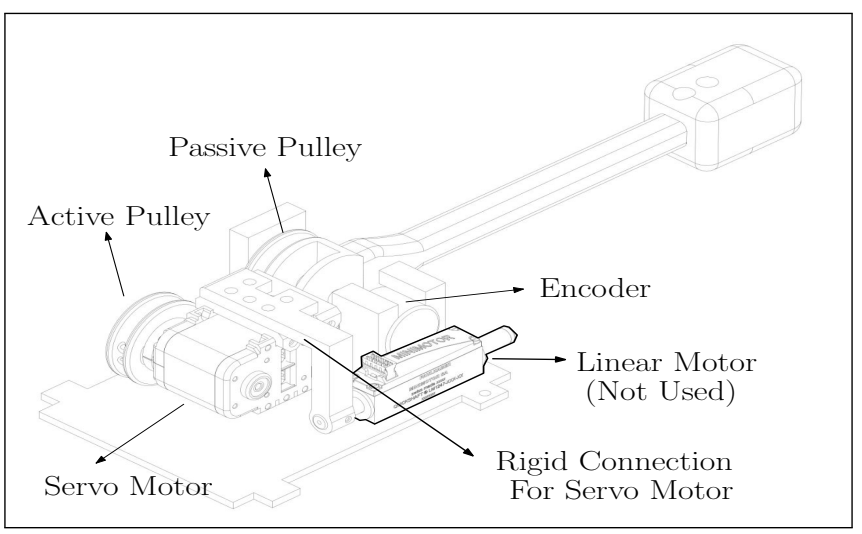

Fig. 3: CAD Sketch of the flexible-joint arm.

flexible-joint arm is controlled via an unconstrained optimal control strategy to maximize the rigid link velocity by controlling the servo motor velocity and using the potential energy stored on the elastic components. The quadrotor is controlled using near hovering algorithm, which is an effective way of controlling quadrotor state, while it can also be used easily by a human operator for teleoperation tasks. Section IV gives detailed information about the hardware used in flexible-joint arm design, and quadrotor itself. In the same section we explain the experimental setup, and present the results of this study. Finally in Section V we discuss the results of this paper, give useful remarks, and propose future developments on the current design.

\section{DESIGN, MODELING, AND IDENTIFICATION}

In this section we present the design of the flexible-joint arm in detail, from the CAD model to the actual product. Afterwards we derive the mathematical model of the system, and find the physical parameters in this model by using experimental data with system identification methods. The identified model, together with real measurements, is then exploited for evaluating the evolution of the system.

\section{A. Design of the Flexible-joint Arm}

The flexible-joint arm consists of several parts. The rigid parts, with the exception of the actuators, are CAD modeled and 3D printed. As can be seen in Figs. 2 and 3, a rigid pulley is attached to the shaft of the servo motor, which is connected to a second rigid pulley via two elastic elements (see also Fig. 1). The first pulley is referred to as active pulley and the second one as passive pulley. The passive pulley is attached to a rigid link, whose objective is to interact with the

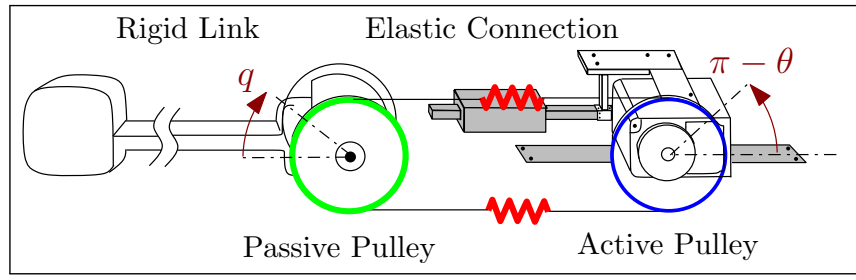

Fig. 4: Schematic figure of the flexible-joint arm with notations used in the paper. The linear motor is grayed out and inactive for the current design. A future development includes activating this part too, for upgrading the current elastic actuator design to a variable stiffness actuator one.

\begin{tabular}{l||l|l|l} 
Link Inertia & $M$ & 0.0019 & $\mathrm{~kg} . \mathrm{m}^{2}$ \\
\hline Spring Constant & $K_{J}$ & 0.3374 & $\mathrm{Nm} / \mathrm{rad}$ \\
\hline Natural Frequency & $\omega_{n}$ & 13.3 & $\mathrm{rad} / \mathrm{s}$ \\
\hline Motor-side Dissipation & $D_{\theta}$ & 0.0364 & N.s $/ \mathrm{rad}$ \\
\hline Link-side Dissipation & $D_{q}$ & 0.0048 & N.s $/ \mathrm{rad}$
\end{tabular}

TABLE I: Estimated parameters of the flexible-joint arm

environment. The position of the active pulley is measured by the encoder of the servo motor. The measurements for the arm motion are collected with a magnetic encoder attached to the passive pulley. The magnet is placed on a cylindric part attached to the center of the passive pulley, and the encoder is placed on a fixed surface.

A second linear servo motor can let the active pulley slide along the bottom surface in order to regulate the distance between the two pulleys. At present however this feature is not used and the distance has been fixed with a rigid connection. The reason for introducing a second linear motor in the design is to have room for future improvements, such as changing the design from an elastic actuator to a variable stiffness actuator by using, e.g., nonlinear elastic components [13]. Finally, a rigid box covering the mechanism except the rigid arm is adopted for protection of the hardware components.

The elastic components between two rigid pulleys are chosen as linear springs (see in Figs. 1 and 4). The two springs are used as antagonistic pairs, i.e., in case one of the springs contracts, the other one relaxes, and viceversa. This is natural way of designing a flexible arm, similar to biceps and triceps in the human arm; where the muscels cannot push, but only pull [13]. The advantage of using antagonistic pairs is that, they allow the full elastic behavior in both directions of the rotation.

Besides the mechanics of the system, the electronics and communication with the arm is another part of the design. This is explained in SectionIV-A in detail.

\section{B. Dynamic Model and Identification}

The physical parameters of the flexible arm are mostly unknown. In this part of the paper we derive these parameters that will be later used for controlling the flexible-joint arm motion. Additionally, we derive the models of the link and of the motor that will be exploited for evaluating the dynamic behavior of the system during fast motions.

The flexible-joint arm consists of motor-side and link 


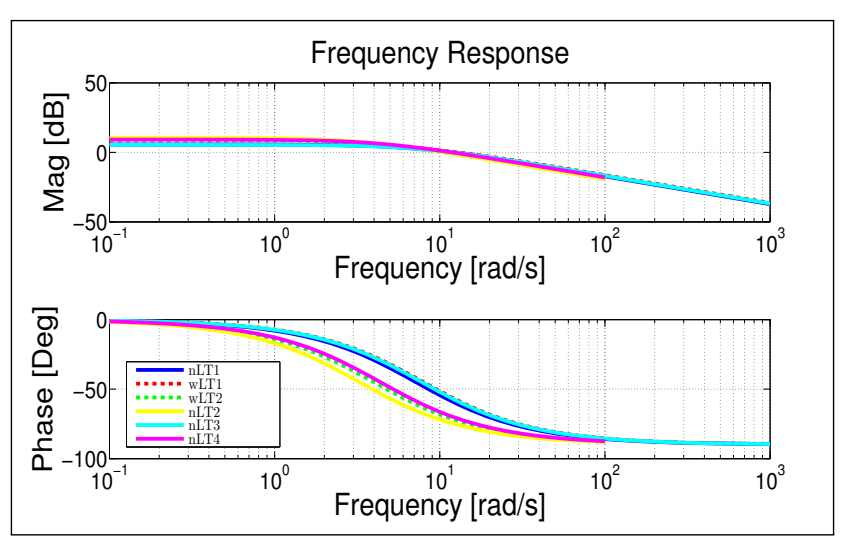

Fig. 5: Frequency response of motor for different step inputs. The input is desired motor velocity, and output is measured motor velocity. The dashed lines represent the conditions 'with load' (wL), and the solid lines the ones with 'no load' (nL). Different colors correspond to pulse trains of different frequencies (T1, T2, T3, and T4).

side dynamics. Both are of second order as shown in [14]. However, if the motor dynamics is considerably faster than the link dynamics, it is possible to assume that the motor velocity can be controlled instantaneously [14], [15]. This means that the servo motor behaves as a perfect velocity source. Under this assumption, the linear dynamics of the flexible-joint arm can be described as

$$
\begin{aligned}
M \ddot{q}+D_{q} \dot{q}+K_{J} q & =D_{\theta} \dot{\theta}+K_{J} \theta \\
\theta & =\int_{0}^{t} \dot{\theta}_{d} d t+\theta_{0}
\end{aligned}
$$

where $\theta \in \mathbb{R}$ and $q \in \mathbb{R}$ are motor side and link side positions, respectively, $M>0$ is the inertia of the rigid link, $K_{J}>0$ is the linear spring constant, and $D_{\theta}>0$ and $D_{q}>0$ represent the dissipations (viscous elements) of the motor side and link side, respectively. The desired motor velocity is shown with $\dot{\theta}_{d}$, and $\theta_{0}$ is the initial motor position.

The validity of the assumption of considering motor as a velocity source depends on the load attached to the motor. Since we are introducing a novel design, it is in our interest to see whether the servo motor can be used as velocity source when the elastic and rigid parts are also attached.

Considering as input the desired motor velocity $\dot{\theta}_{d}$, and as output the measured motor velocity $\dot{\theta}$, the transfer function, in the Laplace domain, is expected to be of the first order, namely

$$
\frac{\dot{\Theta}(s)}{\dot{\Theta}_{d}(s)}=e^{-s \tau} \frac{a}{s+b} .
$$

where $\tau$ represents the system delay.

In order to validate the model (3) and to estimate the parameters $a, b$, and $\tau$ we performed some experiments and used the non-linear least squares method [16]. In Fig. 5 the frequency response of the motor is shown for different step inputs as desired velocity profile and with different conditions: loaded and unloaded case. The output is the measured motor velocity that is retrieved using the motor

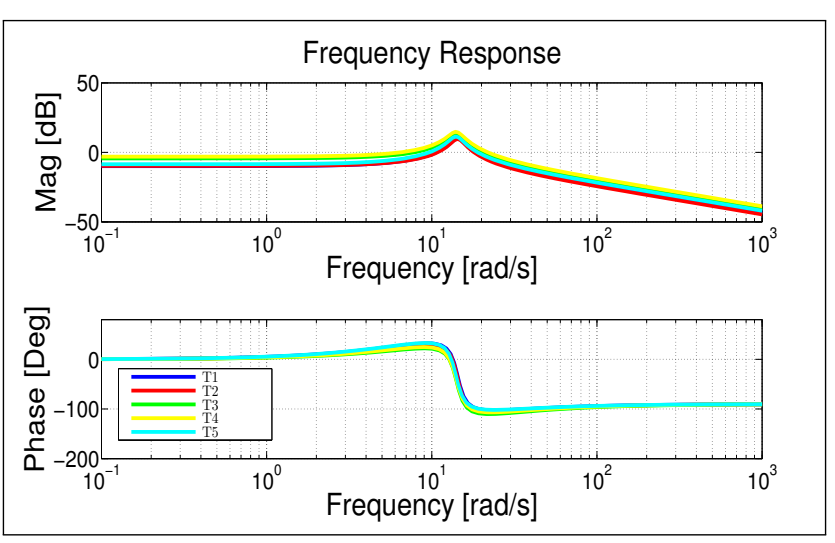

Fig. 6: Frequency response of the flexible-joint arm system for different step inputs. The input is the motor position and the output is the measured link position. Different colors present the trials with different step input profiles in motor velocities.

encoder. The different plot colors correspond to pulse trains of different frequencies. Solid lines are corresponding to conditions where no load is attached to the motor. Dashed lines represent the cases when the motor is connected to the arm though the elastic interconnection. As it can be seen, the frequency response of the system stays almost the same in the frequency range of interest, which is around the natural frequency of the total system (this will be identified in a next step). Hence we can consider the motor as velocity source even when it is connected to its load. A good fit for (3) considering different conditions is found for the parameters $a=13.79$ and $b=7.175$, and $\tau=0.2 \mathrm{~s}$.

The parameters of the flexible-joint arm given in (1) have to be estimated as well. Let us first write the transfer function of the system dynamics for motor position $\theta$ as input and link position $q$ as output. Denoting with $\omega_{n}$ and $G$ the natural frequency and the low-frequency gain of the system, respectively, we obtain:

$$
\frac{Q(s)}{\Theta(s)}=e^{-s \tau} K \frac{s+\mu}{s^{2}+2 \xi \omega_{n}+\omega_{n}^{2}}
$$

where $K=G D_{\theta} / M, \mu=K_{J} / D_{\theta}, \omega_{n}=\sqrt{K_{J} / M}$, and $\xi=D_{q} / 2 \sqrt{K_{J} M}$.

The moment of inertia for the rigid link is computed as $M=0.0019 \mathrm{~kg} \cdot \mathrm{m}^{2}$ from the CAD model shown in Fig. 2. Despite the inertia is easy to compute from the geometry of the system, the dissipative parameters such as damping and frictions are hard to retrieve from a simulation. For this reason, we used non-linear least squares [16] to identify the system parameters. By choosing motor position $\theta$ as input and link position $q$ as output we computed the frequency response of the system for different step input profiles. The results are presented in Fig. 6. The best fit for (4) is

$$
\frac{Q(s)}{\Theta(s)}=e^{-0.2 s} 8 \frac{s+9.2634}{s^{2}+2.573 s+197.3} .
$$

The physical parameters corresponding to this transfer function are shown in Table I.

In Figs. 5 and 6 it is clear that the both motor and link can be operated around the natural frequency of the flexible- 


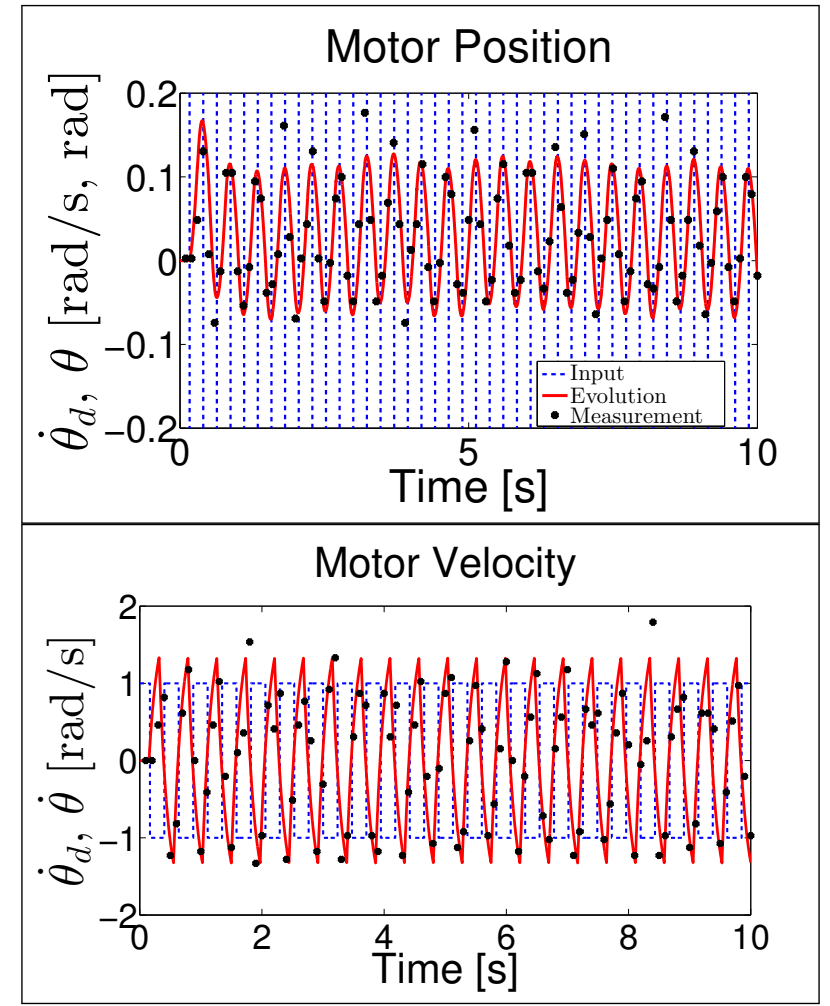

Fig. 7: Motor position and velocities. Blue dashed line represents the optimal control input computed using (9) for $t_{f}=3 \mathrm{~s}$ and $\omega_{n}=13.3 \mathrm{rad} / \mathrm{s}$. Black dots are the measured motor position and velocities using embedded servo motor encoder in $10 \mathrm{~Hz}$. Red plot are for the corresponding evolutions of the identified transfer functions.

joint arm, $\omega_{n}$, when the servo motor is considered as a perfect velocity source. The natural frequency of the system is especially interesting for us, since we would like to test the new design during fast movement tasks, as will be explained in the next section in detail.

Now, by considering the flexible-joint arm as a secondorder LTI system, where desired velocity is input, and motor position and link position are outputs, we have

$$
\frac{\Theta(s)}{\dot{\Theta}_{d}(s)}=e^{-s \tau} \frac{a}{s(s+b)} \text {. }
$$

and

$$
\frac{Q(s)}{\dot{\Theta}_{d}(s)}=\frac{e^{-s \tau}(K a s+K \mu a)}{s^{4}+\left(2 \xi \omega_{n}+b\right) s^{3}+\left(\omega_{n}^{2}+2 \xi \omega_{n} b\right) s^{2}+\omega_{n}^{2} b s}
$$

where both (6) and (7) can be used to evaluate motor and link motions during explosive movements of the system.

\section{CONTROL}

\section{A. Control of the Flexible-joint Arm}

Due to its compliance, the flexible-joint arm is intrinsically safe and an unforeseen interaction with the environment is absorbed by the arm and it perturbs only slightly the motion of the aerial vehicle it is mounted on. Another interesting interactive feature of flexible-joint arms is the possibility of achieving fast, or even explosive motions. Such movements

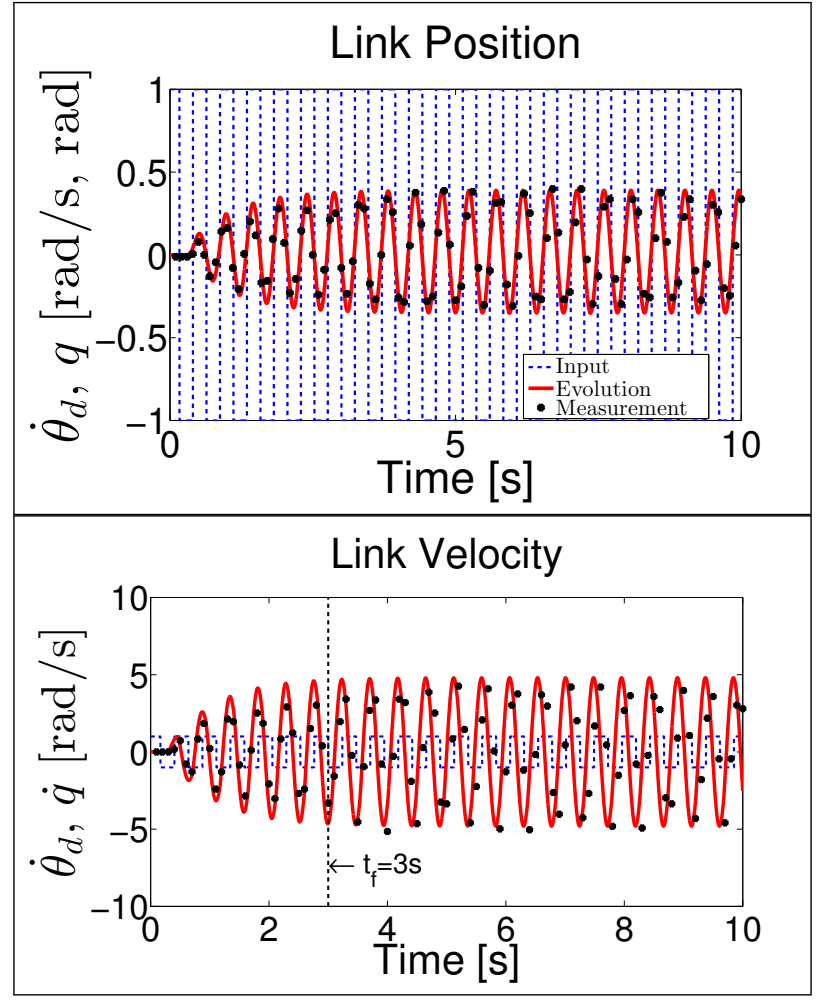

Fig. 8: Link position and velocities. Blue dashed line represents the optimal control input computed using (9) for $t_{f}=3 \mathrm{~s}$ and $\omega_{n}=13.3 \mathrm{rad} / \mathrm{s}$. Black dots are the measured link position and velocities using the magnetic encoder in $10 \mathrm{~Hz}$. Red plots are for the corresponding evolutions of the identified transfer functions.

can be transformed to hammering or throwing tasks using flying robot, which can be useful especially for construction works in relatively high buildings where a ground robot cannot reach.

By explosive movement, we mean the amplification of link velocity using elastic components of the flexible-joint arm. A rigid arm, directly connected to the actuator without any elastic element, will always have the velocity of the motor. However, a flexible-joint arm can reach higher velocities than actuator can provide, thanks to its elastic components. In the following, we first use an unconstrained optimal control strategy for maximizing the link velocity [15]. Afterwards, the trajectory controller of the flying platform is introduced, which is presented before in [17].

1) Maximizing Velocity of the Flexible-joint Arm: It is shown in the literature [15] that the elastic components, e.g., springs, can be used to amplify the velocity of the actuation source. In this paper we will use unconstrained optimal control strategy to maximize the flexible-joint arm velocity in a specified final time $t_{f}$. Hence the cost function to be maximized is

$$
J=\dot{q}\left(t_{f}\right)
$$

Since we found in the previous section the damping factors relatively low, we will neglect them in this paper. Hence, the optimal control policy presented in [14], [15] for unconstrained undamped mass-spring system is suitable for our 


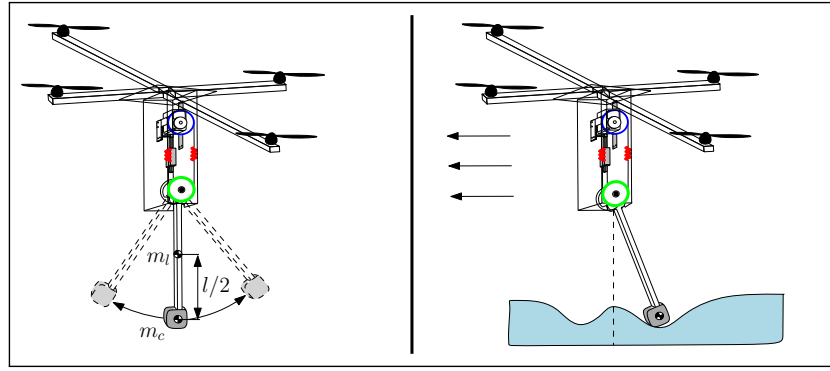

Fig. 9: Flexible-joint arm is attached to the quadrotor system. Two experimets are designed; explosive arm movement (on the left) and aerial physical interaction (on the right). The second one is a task, where flexible-joint arm is sliding on a sloped surface, while quadrotor is performing a stable flight. The rigid link mechanism consists of a mass $m_{c}=44 \mathrm{gr}$ at the tip of the link, in a distance of $l=18 \mathrm{~cm}$ to the link center of gravity, where $m_{l}=44 \mathrm{gr}$.

purpose. The optimal controller is then

$$
u^{*}= \begin{cases}\dot{\theta}_{\text {max }}, & \sin \left(\omega_{n}\left(t_{f}-t\right)\right)>0 \\ \dot{\theta}_{\text {min }}, & \sin \left(\omega_{n}\left(t_{f}-t\right)\right)<0\end{cases}
$$

where the optimal control input is $u^{*}=\dot{\theta}_{d}^{*}$. Note that we consider the servo motor as perfect velocity source (see Section II) which is however constrained, i.e., $u^{*} \in$ $\left[\dot{\theta}_{\min }, \dot{\theta}_{\max }\right]$. This type of controller is called bang-bang controller. In our case no state constraints are considered.

We have applied this controller to the system, on board of a quadrotor, by choosing $t_{f}=3 \mathrm{~s}$. The results are shown in Figs. 7 and 8. The control input as desired motor velocity is shown in both figures with blue dashed line. The measurements are depicted with black dots, in $10 \mathrm{~Hz}$ from both motor and link encoders. Here we also evaluated the evolution of the identified transfer functions in $1 \mathrm{KHz}$, which is presented with solid red plots. As seen in the lower plot of Fig. 8 the maximum link velocity is reached in around $t_{f}=3 \mathrm{~s}$, which is almost four times more than the servo motor velocity.

\section{B. Control of the Aerial Platform}

In this part we give an overview of the controller used for quadrotor, to achieve a stable flight while the flexible-joint arm is operating. In addition, we expect from the controller to give a good tracking results when a human operator is controlling its trajectory. In real-life cases, where flying robots are required to be interacting with their environment (such as in out-door scenarios) we believe that controlling the flying system with human input is as important as fully autonomous trajectory control. For this reason, we choose to control the quadrotor using so called near hovering control, as deeply explained in [17].

Briefly, the controller is developed for teleoperation tasks using quadrotors, where it allows operator more focusing on high-level tasks, while low-level ones are hidden from the operator such as underactuation of the translational dynamics. The goal of the controller is to make quadrotor follow a smooth trajectory in translational motion, using the inputs given by the human operator, while trying to keep hovering

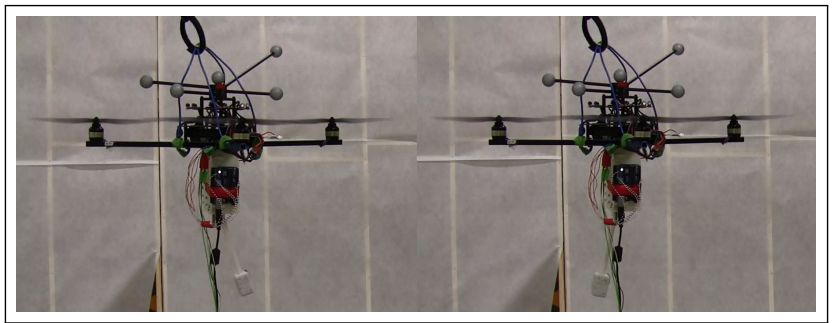

Fig. 10: Explosive movement of the flexible arm during flight on the quadrotor. On the left picture arm swings right, and on the right picture it swings left. More detail is given in the video attached to this paper.

configuration, i.e., small pitch and roll angles, as much as possible. In this paper we used this controller for controlling the translational trajectory of the quadrotor, where the novel flexible-joint arm is attached to its center of gravity as shown in Fig. 9.

\section{HARDWARE AND EXPERIMENTS}

The flexible-joint arm is a combination of 3D printed rigid parts, elastic components, actuator, and measurement units with their electronics. The 3D printed parts and elastic elements are introduced previously in detail. In this section we will present the actuator, i.e., the servo motor, the measurement units, and the electronics. Moreover, we will briefly give information about the flying platform and the setup for the experiments. Finally we present the experimental results.

\section{A. Hardware of the Flexible-joint Arm}

The main limitation of the design is the weight and size of the flexible-joint arm, since it is planned to be mounted on a small-scale quadrotor. For this reason we chose Dynamixel AX-12A servo motor, which is both velocity and position controllable. The motor provides velocity, position, load, and temperature measurements. The communication with servo motor is done using serial communication block of Simulink-Matlab, which is running in $10 \mathrm{~Hz}$ [18]. The rest of the simulink file is running in $1 \mathrm{KHz}$, which includes the controller of the flexible-joint arm. The position and velocity measurements of the passive pulley (so the rigid link) are acquired using 10-bit AEAT-6010 magnetic encoder $^{1}$. The encoder is fixed to the body of the actuator, whereas the rotating part (magnet) is directly connected to the passive pulley. The encoder readings are acquired using serial channel of Arduino-Uno ${ }^{2}$, and transferred to another serial communication block in the same simulink file where the servo motor is controlled. The encoder measurements are also done in $10 \mathrm{~Hz}$. Simulink file was running under Windows- 8 machine using Matlab-2013b.

\section{B. Experimental Setup}

The experiments are conducted on the flying platform, which is a quadrotor $\mathrm{UAV}^{3}$. The maximum payload of the

\footnotetext{
${ }^{1}$ http://www.avagotech.com/docs/AV02-0188EN

${ }^{2} \mathrm{http} / / /$ arduino.cc/en/Main/arduinoBoardUno

${ }^{3}$ https://www.mikrocontroller.com
} 


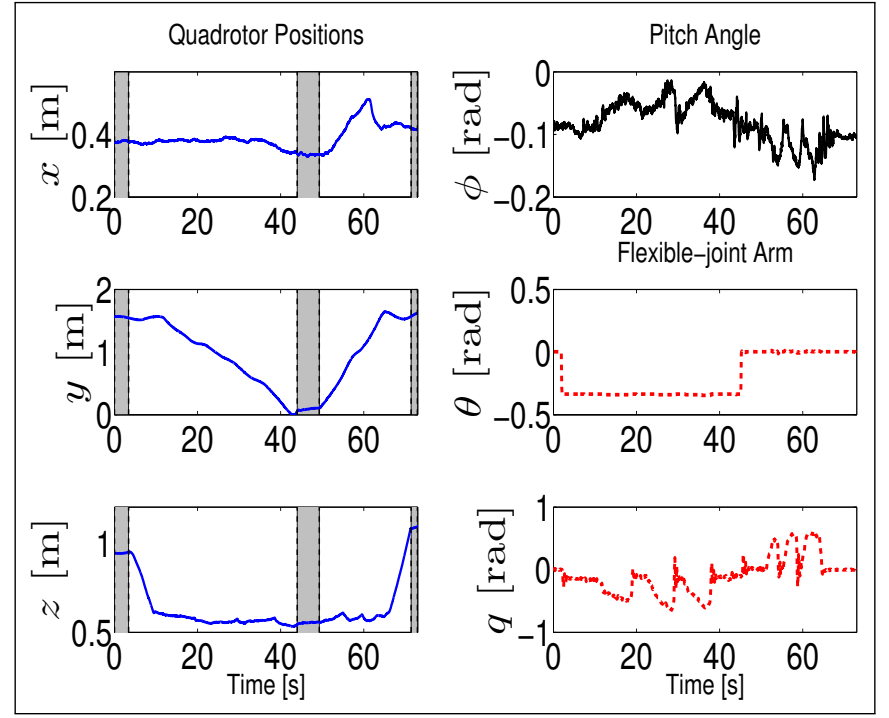

Fig. 11: Results of aerial physical interaction with flexible-joint arm. On the left side three Cartesian coordinates are figured (blue solid lines) where the grayed areas are representing the hovering case of the quadrotor. Black solid figure on the right shows the pitch angles, and red dashed plots are measurements of motor angles $(\theta)$ and link angles $(q)$ for the flexible arm during the flight.

quadrotor is about $2 \mathrm{~kg}$, hence we designed the flexiblejoint arm as light as possible (total mass of the flexiblejoint arm is $360 \mathrm{gr}$ ). The maximum reachable rotation angle of the flexible-joint arm is limited to $q \in[-1,0.55]$ rad. The experiments are done in-door, and we use a motion capture system (MoCap to have good estimation of the quadrotor position. On board of the quadrotor there are Inertial Measurement Unit (IMU), flight controller, brushless motor controller, battery and markers for the MoCap. The total system weight is 1360 gr. The communication with onboard electronics of the quadrotor and the MoCap is done on an Ubuntu 12.04 machine, using ROS-fuerte [19] and Telekyb software, which is developed for controlling the aerial robots [20].

The quadrotor is controlled by a human operator, using a standard joypad connected to the Ubuntu machine. Besides the operator, another person was holding the security stick (see the videos attached), which is connected to the quadrotor from top. The connection is done with ropes for security reasons, which have no tension the during flight.

\section{Experiments and Results}

In this paper we designed two experiments; fast movement of the arm, and stable aerial physical interaction task (see Fig. 9).

For the first experiment, the quadrotor is actively keeping a hovering condition, and the optimal controller presented in (9) is applied to the flexible arm. The motion of the arm is represented in Fig. 10, which is a snapshot of the real experiment. The results are given in Fig. 8, where the link velocity is clearly amplified compared to desired and measured servo motor velocities given in Fig. 7. Thanks to the storage of elastic energy due to the flexible joint, the

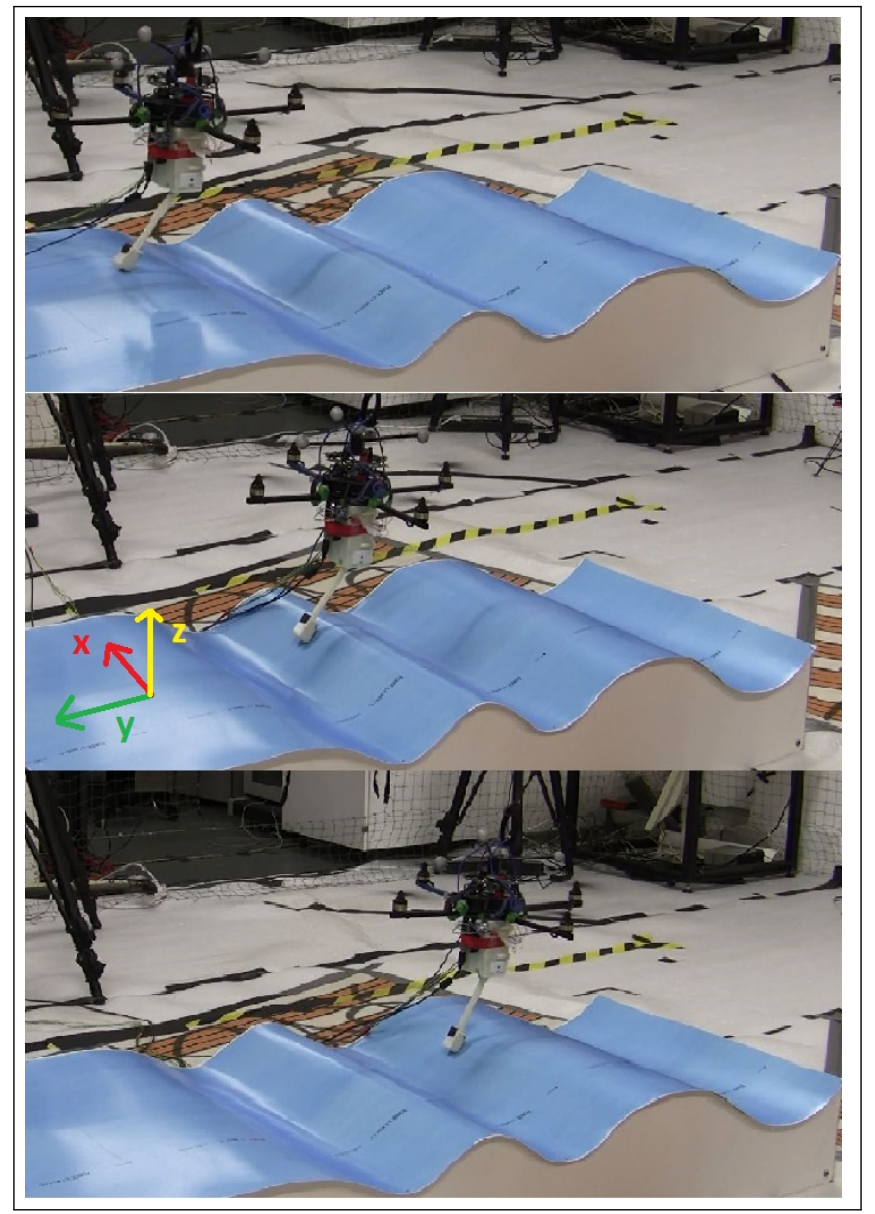

Fig. 12: Experiments for aerial physical interaction. Three figures represent the evolution of the motion along the $-\hat{y}$-axis, as mentioned in Fig. 11, where the results are shown. Three axes of Cartesian coordinates are shown in the middle figure in three different colors. Full experiment is available in the video attached to this paper.

motion of the link is amplified and this is the biggest step to take towards the implementation of explosive motions. Once the motion amplification is achieved, it is only necessary to endow the arm with a proper tool for achieving the desired explosive task (e.g., aerial hammering or throwing).

The second experiment is for the aerial physical interaction task using flexible-joint arm on the quadrotor. A platform with a slopped surface (blue colored surface in Fig. 12) is designed for this experiment. The goal is to have a stable flight, while quadrotor equipped with the arm is sliding on the blue surface in safe. For this experiment, we have set the proportional gain of the position controller to zero for near-hovering controller along the $\vec{y}$-axis [17]. The reason is to let the quadrotor drift along the direction of motion, while the flexible-joint arm is interacting with the environment (see Fig. 12). The flexible-joint arm is position-controlled in this case, meaning that we send the desired motor position and velocity values to a set value at will. The results are given in Fig. 11. Left side of the figure shows the positions of the quadrotor in three Cartesian axes, with blue solid lines. The gray areas are representing the hovering condition of 
the quadrotor, meaning that its position is fixed. After the first grayed area, quadrotor starts to descend and approaches to the blue platform so that the flexible arm touches the surface. Later the quadrotor flies along the $-\vec{y}$-axis while trying to keep contact with the sloped surface (see Fig. 12). After holding in hovering position in the second grayed area, it flies back along the $+\vec{y}$ direction by keeping the contact. Finally it arrives to initial position, ascends, and stays in hovering condition as in the beginning. The pitch angles (rotation along $\vec{y}$ axis) are given as black solid lines in Fig. 11. The near hovering controller is keeping the system and the interaction stable during the whole flight. The red plots in the same figure are showing the motor positions $(\theta)$, and the link positions $(q)$ of the joint arm. Depending on the direction of the flight, different motor positions are set to the servo motor. The changes in the link position is clearly seen in the figure, which is following the profile of the interaction surface. The motor positions, on the other hand, are fixed unless it is not commanded. Thanks to the flexibility of the joint the arm can safely interact with an unknown environment. Furthermore, since the elasticity of the joint absorbs the impact with the environment, the motion of the UAV is left almost unperturbed by the unforeseen impact with the environment.

We encourage the reader to see the video attached to this paper, where we present these two experiments in detail. In addition, two preliminary hammering tests have been shown, using flexible-joint arm attached on a flying quadrotor. In the first hammering experiment, flexible-joint arm is controlled by a standart joystick, while in the second one we use leapmotion sensor ${ }^{4}$ to operate the arm based on human hand motion, using ROS-groovy interface.

\section{CONCLUSIONS}

In this paper we presented a novel light weight flexiblejoint arm for light-weight flying robots, to be used in aerial physical interaction tasks. By using system identification methods, the physical parameters of the new design are estimated and presented. An optimal controller to maximize the elastic link velocity is used to perform fast motion tasks. Such design is not only limited to explosive interaction tasks, but can also be used to achieve stable contact with the environment thanks to its elastic design. The design and the controller are tested on a flying quadrotor and validated with experimental results.

A next step is to improve this design to variable stiffness actuator (VSA), and perform various aerial physical interaction tasks together with a flying system using specific controllers for aerial physical interaction. In fact, the flexiblejoint arm proposed in the paper can be extended to a variable stiffness joint arm, by adding a linear motor and changing elastic components from linear springs to nonlinear ones, such as using rubbers.

\footnotetext{
${ }^{4}$ https://www.leapmotion.com/
}

\section{REFERENCES}

[1] B. Yüksel, C. Secchi, H. H. Bülthoff, and A. Franchi, "Reshaping the physical properties of a quadrotor through IDA-PBC and its application to aerial physical interaction," in 2014 IEEE Int. Conf. on Robotics and Automation, Hong Kong, China, May. 2014, pp. 62586265.

[2] — , "A nonlinear force observer for quadrotors and application to physical interactive tasks," in 2014 IEEE/ASME Int. Conf. on Advanced Intelligent Mechatronics, Besançon, France, Jul. 2014, pp. 433-440.

[3] D. J. Lee and C. Ha, "Mechanics and control of quadrotors for tool operation," in 2012 ASME Dynamic Systems and Control Conference, Fort Lauderdale, FL, Oct. 2012.

[4] H. Nguyen and D. Lee, "Hybrid force/motion control and internal dynamics of quadrotors for tool operation," in 2013 IEEE/RSJ Int. Conf. on Intelligent Robots and Systems, Tokyo, Japan, November 2013, pp. 3458-3464.

[5] V. Lippiello and F. Ruggiero, "Exploiting redundancy in cartesian impedance control of UAVs equipped with a robotic arm," in 2012 IEEE/RSJ Int. Conf. on Intelligent Robots and Systems, Vilamoura, Portugal, Oct. 2012, pp. 3768-3773.

[6] F. Forte, R. Naldi, A. Macchelli, and L. Marconi, "Impedance control of an aerial manipulator," in 2012 American Control Conference, Montreal, Canada, June 2012, pp. 3839-3844.

[7] K. Kondak, K. Krieger, A. Albu-Schäffer, M. Schwarzbach, M. Laiacker, I. Maza, A. Rodriguez-Castano, and A. Ollero, "Closed-loop behavior of an autonomous helicopter equipped with a robotic arm for aerial manipulation tasks," International Journal of Advanced Robotic Systems, vol. 10, pp. 1-9, 2013.

[8] ARCAS, "EU Collab. Project ICT-287617," www.arcas-project.eu.

[9] S. Kim, S. Choi, and H. J. Kim, "Aerial manipulation using a quadrotor with a two dof robotic arm," in 2013 IEEE/RSJ Int. Conf. on Intelligent Robots and Systems, Tokyo, Japan, November 2013, pp. 4990-4995.

[10] M. Fumagalli, R. Naldi, A. Macchelli, R. Carloni, S. Stramigioli, and L. Marconi, "Modeling and control of a flying robot for contact inspection," in 2012 IEEE/RSJ Int. Conf. on Intelligent Robots and Systems, Vilamoura, Portugal, Oct 2012, pp. 3532-3537.

[11] G. Gioioso, M. Ryll, D. Prattichizzo, H. H. Bülthoff, and A. Franchi, "Turning a near-hovering controlled quadrotor into a 3D force effector," in 2014 IEEE Int. Conf. on Robotics and Automation, Hong Kong, China, May. 2014, pp. 6278-6284.

[12] D. J. Braun, H. Matthew, and S. Vijayakumar, "Optimal variable stiffness control: formulation and application to explosive movement tasks," Autonomous Robots, vol. 33, no. 3, pp. 237-253, 2012.

[13] R. V. Ham, T. G. Sugar, B. Vanderborght, K. W. Hollander, and D. Lefeber, "Compliant actuator designs," IEEE Robotics \& Automation Magazine, vol. 16, no. 3, pp. 81-94, 2009.

[14] M. C. Ozparpucu and S. Haddadin, "Optimal control for maximizing link velocity of visco-elastic joints," in 2013 IEEE/RSJ Int. Conf. on Intelligent Robots and Systems, Tokyo, Japan, November 2013, pp. 3035-3042.

[15] S. Haddadin and K. Krieger, "On impact decoupling properties of elastic robots and time optimal velocity maximization on joint level," in 2012 IEEE/RSJ Int. Conf. on Intelligent Robots and Systems, Vilamoura, Portugal, October 2012, pp. 5089-5096.

[16] L. Ljung, System Identification: Theory for the User. Prentice Hall, 1986.

[17] D. J. Lee, A. Franchi, H. I. Son, H. H. Bülthoff, and P. Robuffo Giordano, "Semi-autonomous haptic teleoperation control architecture of multiple unmanned aerial vehicles," IEEE/ASME Trans. on Mechatronics, Focused Section on Aerospace Mechatronics, vol. 18, no. 4, pp. 1334-1345, 2013.

[18] A. Mensink, "Characterization and modeling of a dynamixel servo," Report nr. 035CE2008, Control Engineering Dept., University of Twente, Tech. Rep., Nov. 2008.

[19] M. Quigley, K. Conley, B. Gerkey, J. Faust, T. Foote, J. Leibs, R. Wheeler, and A. Y. Ng, "ROS: an open-source Robot Operating System," in Workshop on Open Source Software in Robotics at the 2009 IEEE Int. Conf. on Robotics and Automation, Kobe, Japan, May 2009.

[20] V. Grabe, M. Riedel, H. H. Bülthoff, P. Robuffo Giordano, and A. Franchi, "The TeleKyb framework for a modular and extendible ROS-based quadrotor control," in 6th European Conference on Mobile Robots, Barcelona, Spain, Sep. 2013, pp. 19-25. 